Sharif University of Technology
Scientia Iranica
SCIENTIA
IRAN Transactions B: Mechanical Engineering
www.scientiairanica.com

\title{
Modeling Paramecium swimming in a capillary tube
}

\author{
A.N. Sarvestani, A. Shamloo* and M.T. Ahmadian \\ School of Mechanical Engineering, Sharif University of Technology, Tehran, Iran. \\ Received 7 December 2014; received in revised form 8 February 2015; accepted 31 May 2015
}

KEYWORDS
Paramecium;
Swimming;
Modeling;
Modified boundary
element.

KEYWORDS

Modeling;

element.

\begin{abstract}
In certain types of biomimetic surgery systems, micro robots inspired by Paramecium are designed to swim in a capillary tube for gaining access to internal organs with minimal invasion. Gaining insight into the mechanics of Paramecium swimming in a capillary tube is vital for optimizing the design of such systems. There are two approaches to modeling the physics of micro swimming. In the envelope approach, which is widely accepted by researchers, Paramecium is approximated as a sphere, self-propelled by tangential and normal surface distortions. However, not only is this approach incapable of considering the specific geometry of Paramecium, but it also neglects short range hydrodynamic interactions due to beating cilia, thus, leading to dissimilarity between experimental data and simulation results. The aim of this study is to present a sub layer approach to modeling Paramecium locomotion that is capable of directly applying hydrodynamic interactions due to beating cilia onto the Paramecium boundary. In this approach, the Paramecium boundary is discretized to hydrodynamically independent elements and, at each time step of swimming, a specific function is fit to the Paramecium boundary. Then, element coordinates are extracted and fluid dynamic equations are solved to model the physics of micro swimming.
\end{abstract}

(C) 2016 Sharif University of Technology. All rights reserved.

\section{Introduction}

In flow regimes pertinent to Paramecium locomotion, viscous forces dominate inertial forces; consequently, specific symmetry breaking events are needed to cause successful propulsion [1]. Paramecium propulsion is achieved via coordinated motion of as many as 4000 non-symmetrically moving organelles called cilia [2]. So, accurate simulation of hydrodynamic interactions between cilia is invaluable in determining the mechanism of Paramecium locomotion. Micro swimming requires complex mechanisms of propulsion which cannot be understood by modeling the symmetry breaking events due to beating cilia via a sub layer approach. In the case of micro robots used for minimally invasive medicine, optimization of the locomotion mechanism

*. Corresponding author. Fax: +98 2166165599 E-mail addresses: shamloo@sharif.edu (A. Shamloo); ahmadian@sharif.edu (M.T. Ahmadian) could help reduce energy consumption, which is of great interest among researchers [3].

Attempts to find a mathematical or numerical model for swimming micro organisms date back to the early 1950s. In a pioneering work, Lighthill derived a closed mathematical form for modelling low-Reynolds number locomotion, called the squirmier model [4]. Later, in 1971, Blake did some corrections to the closed form derived by Lighthill and developed the spherical envelope approach for modeling the squirming motion [5]. In this approach, a micro swimmer is modelled as a sphere able to propel itself using wave-like surface deformations. These wave-like surface deformations originate from the coordinated motion of cilia tips called the metachronal wave. The metachronal wave is nonsymmetric as a result of nonsymmetric deformations of beating cilia. The ciliary beat is divided into effective and recovery strokes. The effective stroke is for propelling the fluid in a forward direction, while the recovery stroke, which lasts longer, returns the 
cilium to its initial condition with minimum backward propulsion [6]. Many analytical and numerical studies have been undertaken for improving the accuracy of the squirmer model. For example, one of the most recent contributions to this model is the work of Wang and Ardekani who solved the problem of unsteady squirming motion [7]. Their great contribution to the squirming model made this model capable of considering added mass and rotation along the swimming axis, a feature that is observed in experimental studies [8].

The most efficient stroke of surface deformations for propulsion was characterized by defining an efficiency function for surface deformations and maximizing this function [9]. The specific surface deformation stroke for optimal feeding of micro organisms was characterized by finding the stroke pertinent to the optimal flow of nutrients towards the cell surface [10]. Also, it has been identified that only hydrodynamic interactions cause Paramecium to swim in a helical trajectory in a capillary tube [11].

Another trend in numerical simulation of lowReynolds swimming was inspired by the pioneering work of Gueron and Liron [12]. Due to the development of computers with high computational capacity in the 1990s, this class of model, termed a sublayer model, was implemented by direct measurement of all hydrodynamic interactions due to beating cilia [13]. The problem with this approach is its high computational cost [14].

Although it has been proved that for modeling a densely packed arrangement of cilia, the envelope model is the best approach, this method has some problems with the specification of boundary conditions pertinent to the motion of cilia tips; therefore, it has been unable to correctly predict the maneuvering mechanism of ciliates with densely packed arrangement of cilia. In order to overcome this problem, a modified boundary element method for simulation of Paramecium swimming seems to be appropriate.

In the current work, it is intended to present a modified boundary element method for simulation of Paramecium locomotion in two dimensions. In this approach, Paramecium is discretized to boundary elements which are hydrodynamically independent from each other. In other words, because hydrodynamic interactions in low-Reynolds number regimes are short ranged [15], it is possible to increase element length so that the boundary elements become hydrodynamically independent of each other. As a result, creeping flow equations can be solved for each element independently. This leads to great reductions in computational cost. In the modified boundary element method, instead of considering a wavy surface for Paramecium and specifying boundary conditions pertinent to a travelling wave [16], its membrane, at many time steps, is discretized to boundary elements that contain 5 cilia

beating together. The data for the ciliary beat are extracted from the bio-inspired fluid lab at Virginia Tech, which consist of the coordinates and velocities of each point on Paramecium at each time step (see Figure S2 and associated table in Supplementary Material (Section 6)) [16]. For each boundary element, at each time step, normal and tangential components of viscous forces are extracted via a creeping flow simulation. Integrating the normal and tangential viscous forces on the Paramecium boundary leads to extraction of propulsive force at each time step. This leads to extraction of the swimming velocity at each time step, and, thus, the swimming trajectory can be defined. The swimming trajectory obtained in this study is the result of time dependent simulation of Paramecium swimming in a capillary tube and is in agreement with experimental data, proving that hydrodynamic interactions play a key role during the swimming of Paramecium in a capillary tube.

\section{Modeling cilium}

With regard to previous studies [6], the cilium, at eight time steps of beating (see Figure 1(a)), is modeled as a set of rigid elements. For minimizing computational cost, it is initially needed to find the minimum number of rigid elements for approximation of the cilium's structure with the least possible error. In order

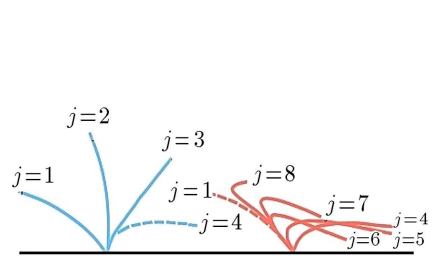

(a)

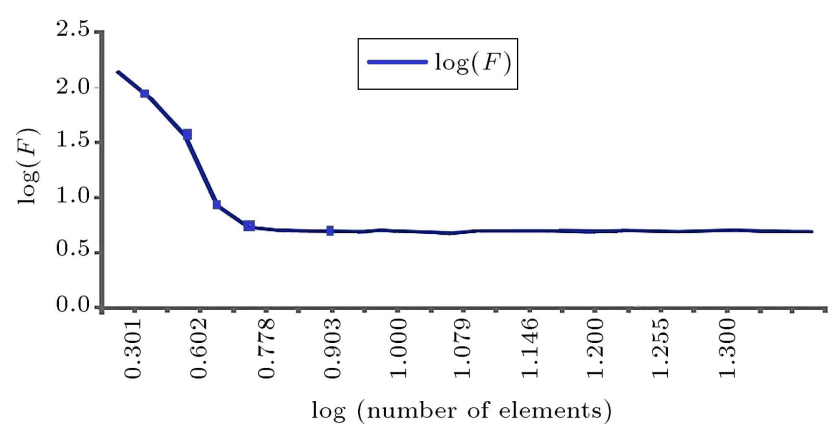

(c)

Figure 1. Modeling each cilium: (a) Cilium geometry at specific time steps. Four of these time steps pertain to the effective, and four of them to the recovery, strokes; (b) $d_{1 j}$, $d_{2 j}$ and $d_{3 j}$ show the normal distance between cilium geometry and rigid elements by which a cilium is approximated; and (c) approximation error in a logarithmic scale, which shows that five rigid elements can lead to an accurate approximation of cilium geometry. 
to quantify the approximation error, each cilium is discretized to sections with similar lengths in 8 time steps (Figure 1(b)). In Figure 1(b), cilium geometry at time step $j$ is discretized to three rigid elements. The approximation error can be characterized by summing the maximum normal distances between cilium sections and the rigid elements, which are shown as $d_{1 j}, d_{2 j}$ and $d_{3 j}$. Initially, it is aimed to find the minimum number of elements which can give the best approximation (i.e. the least approximation error) of the cilium profile. Thus, a function was defined to show the sum of the maximum distances between cilium sections and the approximating elements. This function $(F)$ is numerically quantified using Eq. (1) and plotted in Figure 1(c). This plot shows that for more than five elements, the quantity of function $(F)$ remains unchanged. Considering that computational cost is linearly proportional to the number of these elements, the minimum number of elements that gives an acceptable approximation for modeling cilia (five elements) was chosen for further simulations. The $F$ function is defined as:

$$
F=\sum_{i} \sum_{j} d_{i j}
$$

where $i$ shows the element number, $j$ shows the step number and $d_{i j}$ shows the normal distance between the $i$ th section on the cilium and its corresponding element, in the $j$ th time step of simulation.

\section{Numerical simulation}

In order to characterize different physical parameters of micro swimming, the Boundary Element Method (BEM) is widely used $[17,18]$. According to the methodology introduced by Pozrikidis [19], in the BEM, boundaries are discretized and elements are constructed based on discretization of the boundaries. Lauga and Riley developed a theoretical framework for modeling micro swimming in low Reynolds number flow regimes in complex fluids [20,21]. Their results have been verified by new methods for measuring flow characteristics presented by Valarde et al. [22]. Based on this framework, it has been shown that implementing the boundary element method for modeling the physics of micro swimming is one of the suitable available options [23-25]. In the current work, the Paramecium boundary is discretized to boundary elements containing a specific number of cilia, and fluid equations are solved for each element independently. Modification of the BEM in this study originates from this independence, which is due to the fact that element dimensions are chosen to be so long that hydrodynamic interactions between adjacent elements are negligible.

One of the main challenges in modeling the ciliary beat in Paramecium is determining the element length for discretizing the Paramecium boundary. In fact, it is vital to determine the maximum possible element length, because longer elements lead to lower computational cost.

An important feature in low-Reynolds fluid mechanics is that hydrodynamic interactions are effective at low ranges. Gueron et al. showed that hydrodynamic interactions between two cilia, at a distance of about 1.2 times cilium length, are negligible [12]. Accordingly, it is possible to discretize the Paramecium boundary to elements that are hydrodynamically independent. In other words, we have to find the minimum possible element size for which low-Reynolds equations can be solved independently from other elements. The procedure for determining maximum possible element length is presented in Section 3.2.

Discretizing the Paramecium membrane to hydrodynamically independent elements leads to a considerable decrease in computational cost. In this method, low-Reynolds equations for each element can be solved separately, and the required parameters, such as drag force, can be integrated onto the Paramecium boundary. By integrating the viscous drag on the Paramecium boundary, it is possible to calculate the propulsive force and swimming velocity. After characterizing the element length, a time dependent simulation was conducted in order to determine the swimming trajectory of Paramecium, its speed, and the efficiency of swimming. Simulation results were then compared to experimental data [16] for validating the numerical method. Figure 2(a) shows the flow chart for the numerical simulation used in determining these parameters. The first step in the simulation of each time step of swimming is to read the coordinates and velocities of the cilium at each time step (see Supplementary Material, Figure S2. and associated table (Section 6)). The second step is the construction of boundary elements and determination of their coordinates in the co-moving reference frame. Then, the fluid dynamic solver gives the normal and tangential components of the viscous force, which are integrated over the Paramecium boundary for extraction of the propulsive force and swimming velocity. Finally, in order to combine the different time steps and achieve a time dependent simulation, the swimming velocity calculated during the previous time steps is specified as an initial condition by defining an inlet and an outlet for simulating each boundary element. In other words, if we consider each boundary element on a swimming Paramecium, the fluid passing close to each element could be replaced by an inlet and an outlet (see Section 3.3).

\subsection{Simulation of ciliary beats}

In our simulations, the fluid is regarded as viscous and incompressible, and the filaments that comprise 


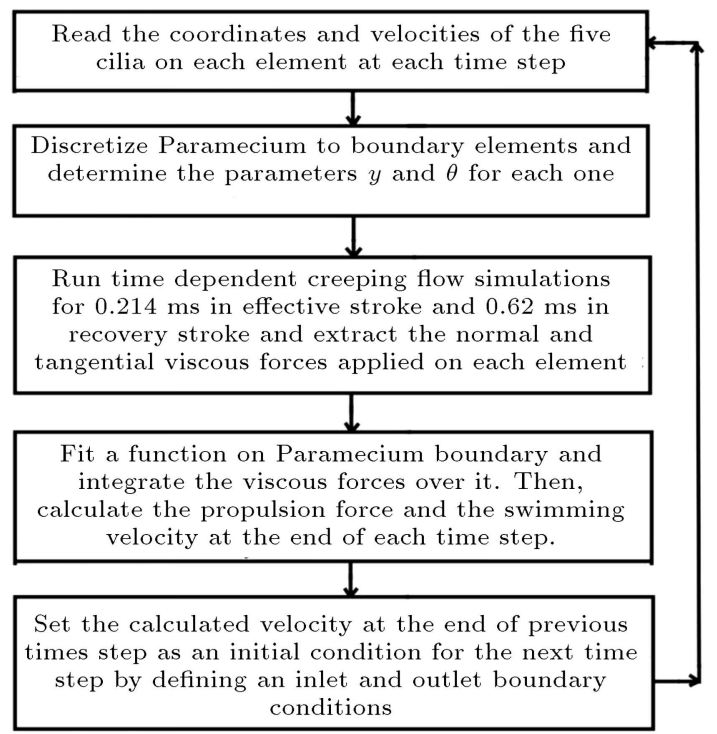

(a)

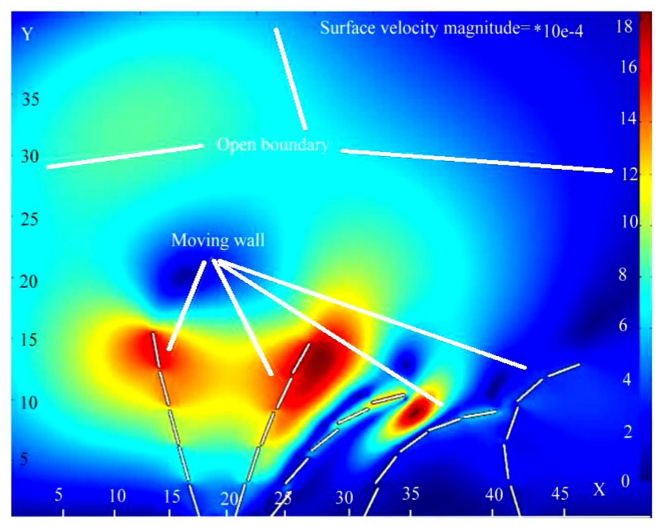

(b)

Figure 2. Numerical simulation: a) The simulation flow chart; and b) fluid velocity distribution at a specific time step of swimming.

nine microtubule pairs of the axoneme are considered rigid boundaries immersed within the fluid. In order to find the velocity and pressure distribution in the fluid domain, Navier-stokes equations for creeping flow are solved. For the case of low-Reynolds swimming, Navier-stokes equations can be simplified to Stokes equations. Eq. (2) shows the momentum and continuity equations in creeping flow:

$$
-\nabla p+\mu \nabla^{2} u=0, \quad \nabla \cdot u=0,
$$

where $\mu$ is fluid viscosity, $u$ represents the velocity vectorized field, and $p$ accounts for the pressure scalar field. After solving the Stokes equations on the fluid domain, we can find the pressure distribution for different fluid nodes. In order to characterize the average hydrodynamic force per length applied onto the cilia as a function of time $(S(t))$, we can integrate the viscous stress $(\sigma)$ over the cilia deformed surface:

$$
\begin{aligned}
& S(t)=\frac{\int \sigma \cdot \hat{n} d s}{L_{\text {cilium }}}, \\
& \sigma=-p I+\mu\left[\nabla u+(\nabla u)^{T}\right],
\end{aligned}
$$

where $p$ is the fluid pressure, $n$ is the unit normal vector to the cilia, $s$ is the length parameter on the moving walls, and $I$ is the identity matrix [11]. Simulation is time dependent by considering the variation of variables with time. Parameters for the mathematical model for Paramecium swimming and their references are presented in Table 1 in the Supplementary Material (Section 6).

In this study, it is required to find the largest amount of time scale that can ensure numerical convergence. If the time scales are chosen too small,

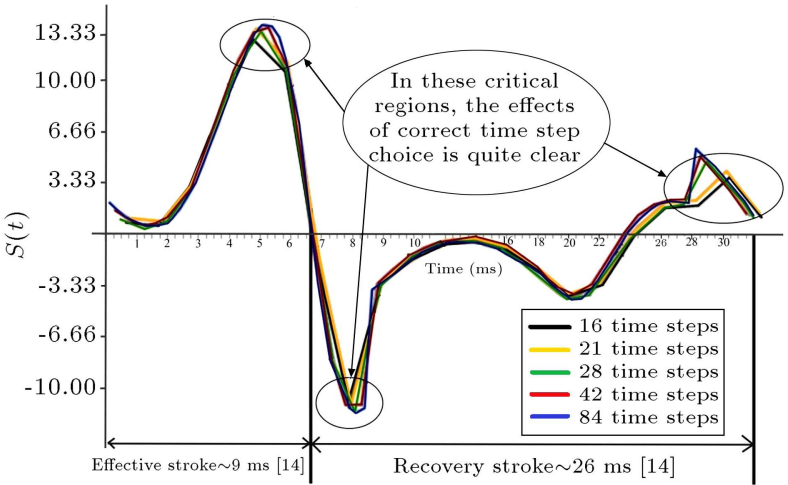

Figure 3. Simulation data (horizontal stress component). These plots show that 84 time steps are enough for convergence in simulation. In other words, in the critical regions around which a circle has been drawn, we see that the plot is not smooth for 21 time steps (the yellow plot); however, the plot is smooth for smaller time steps (84 time steps - the blue plot). This shows the increased chance of convergence for smaller time steps.

the computational cost is increased drastically. Hence, simulations at $16,21,28,42$, and 84 time steps were undertaken, and the plots for the hydrodynamic force per length versus time were obtained.

In Figure 3, the vertical axis shows the average horizontal component of the stress applied to the cilium, which is defined as:

$$
S(t)=\frac{\int \sigma_{x} d s}{l_{c}}
$$

where $\sigma_{x}$ is the horizontal component of the viscous stress applied to the cilium, $d s$ is the length of the element, and $l_{c}$ is equal to the cilium length. According to this figure, the diagrams of total stress 
at different time steps can be considered a measure for the convergence of the simulation. From this figure, it is obvious that at some critical regions of the effective and recovery strokes, the results of simulations at different time steps, in which cilia are located at different positions, are significantly different from each other. The diagram in Figure 3 shows that if we discretize duration of the ciliary beat to 84 time steps, the measured hydrodynamic properties at each time step are so close to the data for the next time step that each time step can be an initial condition for the next time step. This means that 84 time steps can be considered an optimum number for our simulations. In the simulation of objects moving in fluids, the object has different positions at different time steps. If, in the simulation procedure, time is not discretized to small enough steps, the moving object may be at positions far from each other in different time steps. This leads to inaccurate results which may not be in agreement with experimental data.

\subsection{Coordinate system and characterization of element length}

In order to model Paramecium locomotion, it is required to discretize its boundary so that hydrodynamic interactions between discrete elements are negligible. Hydrodynamic interactions between each beating cilium and its neighbors are significant in a finite domain called the cilium influence range. Gueron and Liron. proved that this influence range is 1.2 times cilium length [12]. In this study, this influence range is investigated by measuring the viscous stress applied by each cilium onto its neighbors. In other words, simulations with one to eight beating cilia are conducted and time averaged drag is applied to one of them (the first from the left is measured using Eqs. (3) and (4), and plotted in Figure 4(a)). This figure indicates that hydrodynamic effects are negligible between five adjacent cilia. Because ciliary spacing is $3 \mu \mathrm{m}$ and cilium length is $15 \mu \mathrm{m}$, the cilium influence range is calculated as 1.11 times cilium length. Therefore, according to Figure 4(a) and (b), we chose five cilia on each boundary element.

\subsection{Simulation of ciliary propulsion in Paramecium}

The numerical simulation procedure (Figure 2(a)) begins with the construction of boundary elements and determination of their coordinates in a Cartesian reference frame that is attached to the capillary tube wall (this is the simulation input data which depends on the diameter of the capillary tube and the dimensions of the chosen Paramecium) (see Figure 5). Each

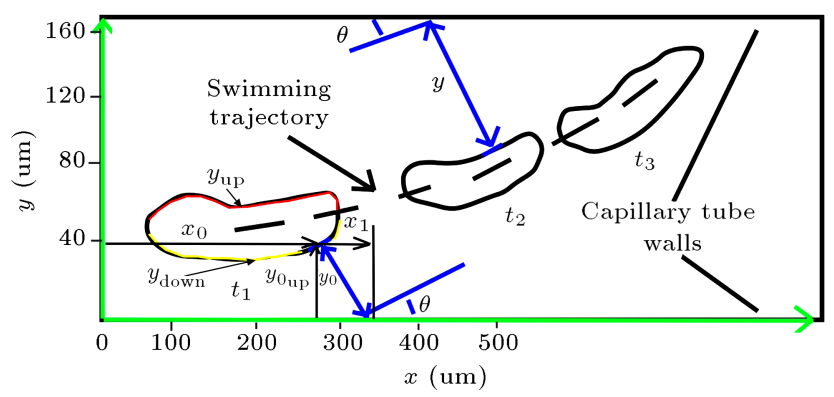

Figure 5. Time dependent simulation of Paramecium swimming. In various time steps of swimming, functions representing the geometry of the Paramecium boundary are extracted. These functions lead to determination of boundary element coordinates at each time step.

Coordinates for boundary elements are then transformed to the co-moving reference frame by defining the two parameters, $Y$ and $\theta$. $Y$ is the normal distance between the element and the corresponding tube wall, and $\theta$ is the angle of rotation of the specific boundary element with respect to its corresponding wall. Parameters, $Y$ and $\theta$, are used to simulate the ciliary beat in each specific element by setting them as input parameters for the COMSOL model pertinent to each element.

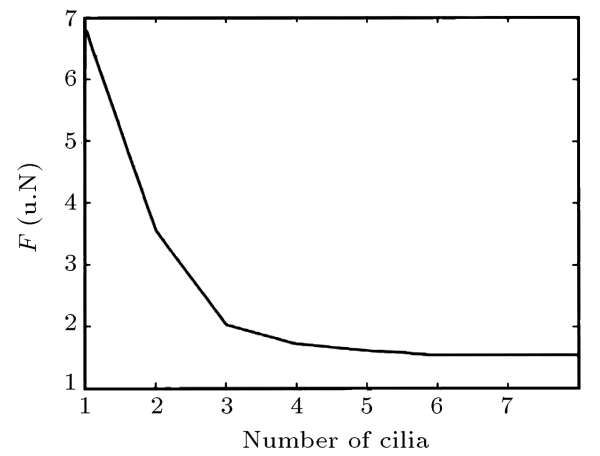

(a)

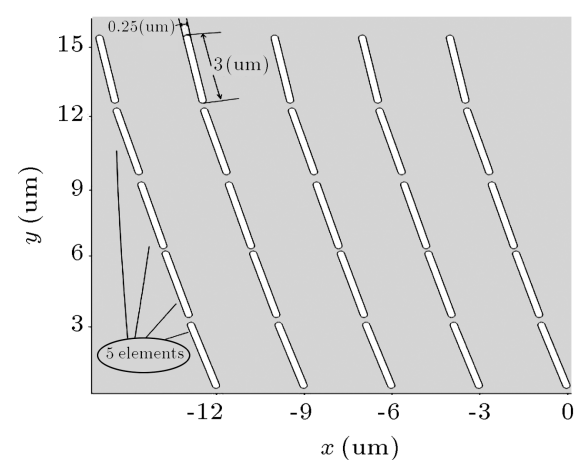

(b)

Figure 4. Methodology for determining the element length: a) In this figure, the viscous force applied on the first cilium from the left is plotted for configurations of one to eight cilia beating together. For configurations of more than five cilia, slight variations in the measured force are observed. In other words, hydrodynamic interactions between the first and fifth cilium are negligible; and b) each element on the Paramecium membrane contains five cilia; consequently, elements are hydrodynamically independent of each other. 
boundary element contains 10340 tetrahedral elements in COMSOL. The chosen solver is the time dependent SPOOLES, which is based on direct LU factorization of the sparse matrix. Direct LU factorization is usually chosen for modeling fluid flow within microfluidics due to its stability and good convergence rate. Indeed, because we intended to simulate Paramecium that changes its geometry while swimming, we should determine the coordinates of different points on its membrane at each time step. Coordinates of different points on the Paramecium membrane are extracted from the video of a swimming Paramecium taken from the bio-inspired lab at Virginia Tech. Also, for investigating propulsive force and swimming velocity, coordinates of different points on its boundary are essential. The swimming velocity is calculated by integrating the function pertinent to the propulsive force and using zero initial conditions.

The next step starts by transferring boundary element coordinates from the Cartesian reference frame $(x, y)$ to the moving reference frame $(Y, \theta)$. For tube diameter $D_{\text {tube }}=200 \mu \mathrm{m}$, at the initial time step $(t=0)$, if we choose a point on the Paramecium's upper boundary with coordinates $\left(x_{0}, Y_{0 \text { up }}\right)$, it can be observed that $34<x_{0}<304 \mu \mathrm{m}$. Then, it is possible to fit function $f\left(x_{0}\right)$ on the upper boundary, so that we have, for each point, $Y_{0 \text { up }}=f\left(x_{0}\right)$ and $\frac{\partial Y_{\text {oup }}}{\partial x}=$ $\frac{\partial f\left(x_{0}\right)}{\partial x}=\dot{f}\left(x_{0}\right)$. In the moving reference frame, $Y$ is the normal distance between each boundary element and its corresponding tube wall. Therefore, in order to calculate the normal distance, the equation for the line normal to an element with coordinates $\left(x_{0}, Y_{0 \text { up }}\right)$ is given in:

$$
y-y_{0}^{\text {up }}=\frac{-d x}{d f\left(x_{0}\right)}\left(x-x_{0}\right) .
$$

for calculating the normal distance, the coordinate of the point at which this line intersects with the upper boundary $\left(x_{1}, 200\right)$ can be calculated from Eq. (7):

$$
-\left(200-f\left(x_{0}\right)\right) \dot{f}\left(x_{0}\right)-x_{0}=x_{1} .
$$

Hence, the normal distance is equal to the distance between the two points, $\left(x_{0}, Y_{0 u p}\right)$ and $\left(x_{1}, 200\right)$, which is given in Eq. (8):

$$
Y=\sqrt{\left(x_{1}-x_{0}\right)^{2}+\left(200-y_{0}^{\mathrm{up}}\right)^{2}} .
$$

The second component in the element coordinates in the moving reference frame is $\theta$, which is characterized from Eq. (9):

$$
\theta_{0}=\cos ^{-1}\left(\frac{200-y_{0}^{\mathrm{up}}}{y_{0}}\right) \text {. }
$$

After adjusting the element coordinates to the moving

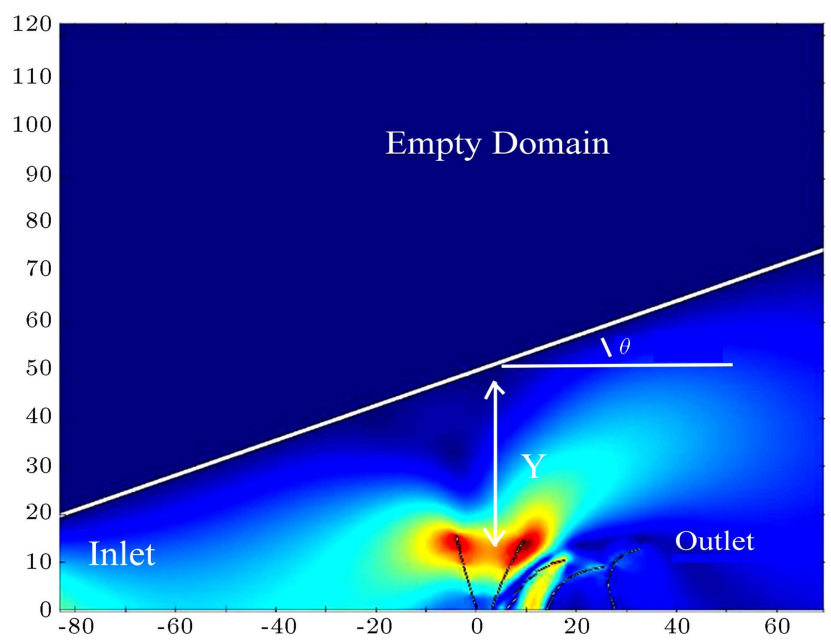

Figure 6. The COMSOL model for extraction of pressure distribution around each boundary element. As previously discussed, boundary elements are hydrodynamically independent; consequently, for each individual element, it is possible to conduct an independent CFD (computational fluid dynamics) analysis by which we can extract the functions pertinent to the normal and tangential components of viscous force on each element, which is specified by special parameters, $Y$ and $\theta$. By integrating these functions over the Paramecium boundary, we can calculate the propulsion force, which could be shown by a set of two time dependent functions; one function for quantity and another for the direction of propulsion force. Also, it may be possible to derive

functions for the propulsion torque, which is of interest for further research.

reference frame, cilia coordinates, and velocities at different time steps, along with transferred coordinates (see Figure S1 and its associated table in the "Supplementary material" (Section 6)), the details of which were extracted from the experimental data provided by the bio-inspired fluid lab at Virginia Tech, [16]), were inserted into the COMSOL model for calculating the viscous force applied onto each boundary element (see Figure 6). Then, functions representing the normal and tangential components of the viscous force as a function of the element coordinates were extracted for calculating the propulsive force and swimming velocity (see Figure S1 in the Supplementary Material (Section 6)). At the beginning of unsteady swimming, the velocity of the organism is equal to zero. For modeling the initial time step of the swimming, the mentioned procedure is accurate enough. However, for modeling swimming at time steps in which the Paramecium has a bulk velocity, the fluid near each boundary element is moving at a specific velocity. The physics of this process could be investigated by defining an inlet and an outlet boundary condition for the COMSOL model (see Figure 6).

For validation of the simulation procedure, the experimental data of Ishikawa and Hota. were 


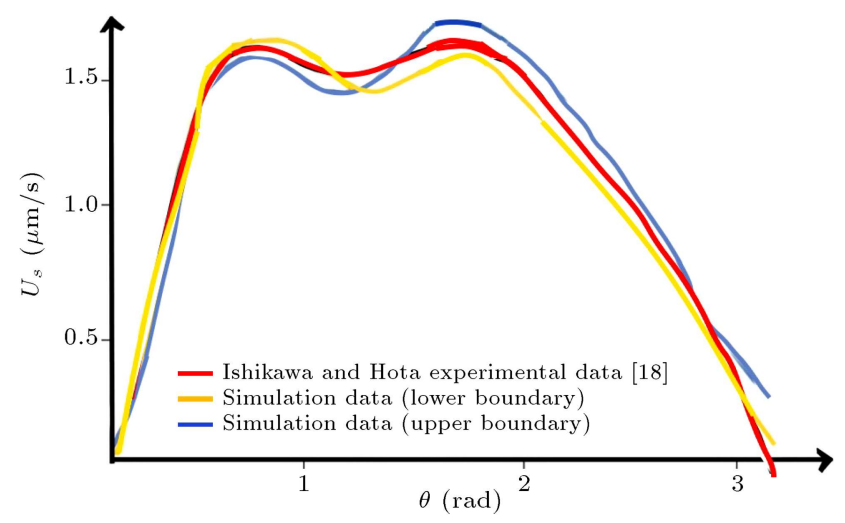

Figure 7. Comparison of the simulation data with experimental data of Ishikawa and Hota [18]. This plot shows the velocity distribution around a swimming Paramecium. Each element of the Paramecium boundary has its unique angle; therefore, the horizontal label depicts the angle of each boundary element with respect to horizontal axes attached to the capillary tube wall.

used [18]. Ishikawa measured the fluid velocity distribution around a swimming Paramecium. We found that our simulation results were in agreement with these data. Figure 7 shows the fluid velocity around a swimming Paramecium versus surface angle. For both simulation and experimental data, fluid velocity limits to zero for the anterior and posterior sections of the swimming Paramecium. For the middle section of the Paramecium, fluid velocity reaches its maximum size. In the middle sections, a slight difference between simulation results and experimental data is observed. This slight difference is due to the fact that they have considered an axisymmetric geometry for a swimming Paramecium, whereas its geometry is not axisymmetric. Their method for obtaining the fluid velocity is particle velocimetry. In this method, the exact geometry of Paramecium could not be taken into consideration and an approximate geometry is investigated.

Also, simulation results of the Paramecium swimming in a capillary tube are compared to Jana's experimental data [16]. These data include swimming velocity, swimming trajectory and swimming efficiency. Discussions about these data are presented in the results and discussions section.

\section{Results and discussions}

Recently, Jana et al. (Bio-inspired fluid lab at Virginia Tech) presented a paper in which they intended to investigate the hydrodynamic effects of confinement on the Paramecium swimming trajectory [16]. For this purpose, they prepared capillary tubes of various diameters and observed the helical trajectory followed by Paramecium swimming in them. Since we are interested in developing a numerical model for explain- ing hydrodynamic effects on Paramecium propulsion, their accurate experimental data were used for our benchmark test.

In order to run the benchmark test, simulations were run for different capillary tube diameters. In the simulation procedure, initially, boundary elements are constructed and their coordinates are extracted. Then, for each time step of swimming, the propulsive force and swimming velocity and trajectory are extracted. Results were compared to Jana's experimental results.

\subsection{Discussions on Paramecium swimming in a capillary tube}

The Paramecium swimming trajectory is a helix which may be characterized by its wavelength and amplitude (see Figure 8). In this work, only a specific section of this helix is extracted. Jana et al. observed that the amplitude of the introduced helix increases nearly linearly with an increase in capillary tube diameter (Figure 8(a) and (b)). Thus, the numerical simulation algorithm can be validated if its results are in good agreement with the experimental data presented by Jana et al. The wavelength and amplitude could be determined by measuring the distance between two points of the wave corresponding to the swimming trajectory, with the slope parallel to the capillary tube wall. According to Figure 8(b), there is good agreement between the experimental data and simulation results.

Figure 8(c) depicts the results of another benchmark test. In this test, swimming velocity, which is non-dimensionalized by the velocity calculated by Blake [5], is measured. In this figure, the black line shows the swimming velocity from the experimental data. From this figure, it is evident that for $R / c<2.1$, simulation and experimental results show increasing trends and converge with each other for very large $R / c$ values [16].

\subsection{Swimming efficiency}

There are various definitions for swimming efficiency. The traditional definition for low-Reynolds swimming efficiency is presented in the following equation. This definition is widely used to determine the most efficient swimming kinematics:

$$
\eta=\frac{<U>T^{*}}{<P>}
$$

In the above equation, $T^{*}$ denotes the force needed to pull a rigid body with similar geometry to Paramecium at constant time-averaged velocity, $\langle U\rangle$, while $\langle P\rangle$ is the mean energy dissipation while pulling the body. In different studies, different methods for calculating $T^{*}$ have been presented. Michelin and Lauga [9] derived $T^{*}=6 \pi\langle U\rangle$ from Stokes' formula for the specific case of a squirmer. However, Jana et al. [16] considered the following equation for calculating $T^{*}$ for the specific case of Paramecium: 


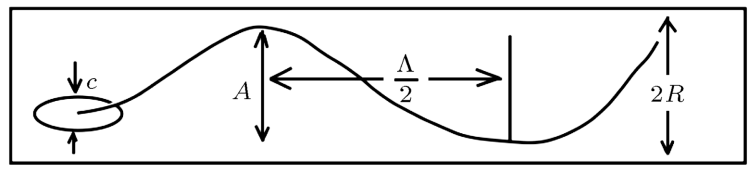

(a)

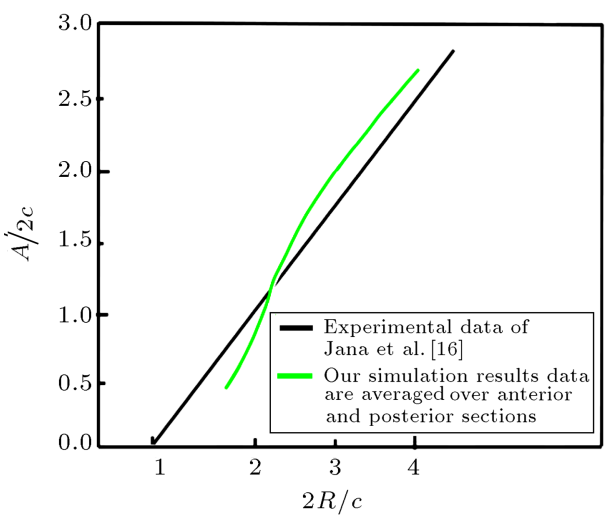

(b)

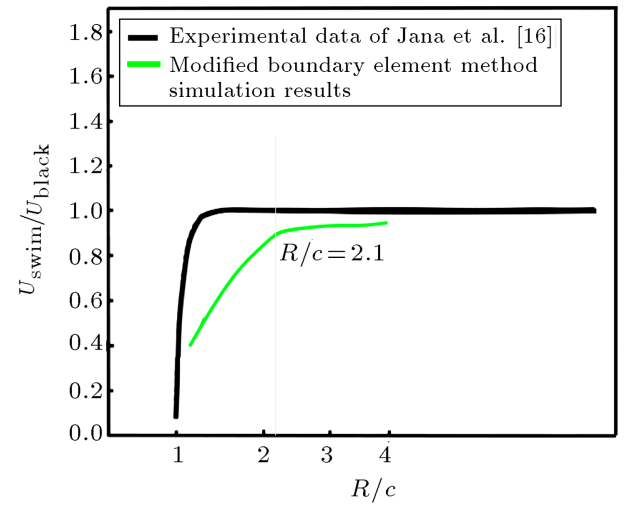

(c)

Figure 8. Time dependent simulation of Paramecium swimming: a) Schematic representation of helical swimming parameters: $c$ is the Paramecium width, $A$ is the amplitude of the swimming trajectory, $R$ is the radius of the capillary tube, and $\Lambda$ is the wave length of the swimming trajectory; b) plot of the non-dimensional amplitude of the swimming trajectory versus the non-dimensional radius of the capillary tube. Simulation data are in agreement with experimental data and show an almost linear increase of amplitude with the increase in capillary tube radius; and c) comparison of results from simulation using a modified boundary element method with experimental data described by Jana et al. [16]. The horizontal axes show the non-dimensional radius of the capillary tube, and vertical axes show the normalized swimming velocity.

$$
T^{*}=\mu \frac{U_{\mathrm{swim}}}{\lambda}
$$

where $\mu$ denotes the fluid viscosity, and $\lambda$ denotes the wavelength of the metachronal wave propagated by cilia. In order to compare our simulation results with the experimental results of Jana et al. [16], we used Eq. (10) for calculating the swimming efficiency.

Figure 9 shows a comparison of Jana et al.'s experimental data [16] with our simulation results. In fact, Jana et al. presented different data for the efficiency of anterior and posterior parts of Paramecium. In our simulation results, we only extracted efficiency for the swimming trajectory of the Paramecium's center of gravity, which is the average of anterior and posterior efficiencies. Simulation results reveal that the swimming efficiency is limited to $4 \%$, while increasing the diameter of the capillary tube in silico. Michelin and Lauga proved that the theoretical efficiency for the squirmer limits to $22.22 \%$ [9]. This is in contrast to our simulation results, indicating that the efficiency of real Paramecium is far different from a squirmer. Thus, the trajectory of a swimming Paramecium may not be calculated by optimizing the efficiency of the squirmer.

\section{Discussion}

In this study, it is primarily intended to develop a sub layer approach for modeling Paramecium locomotion

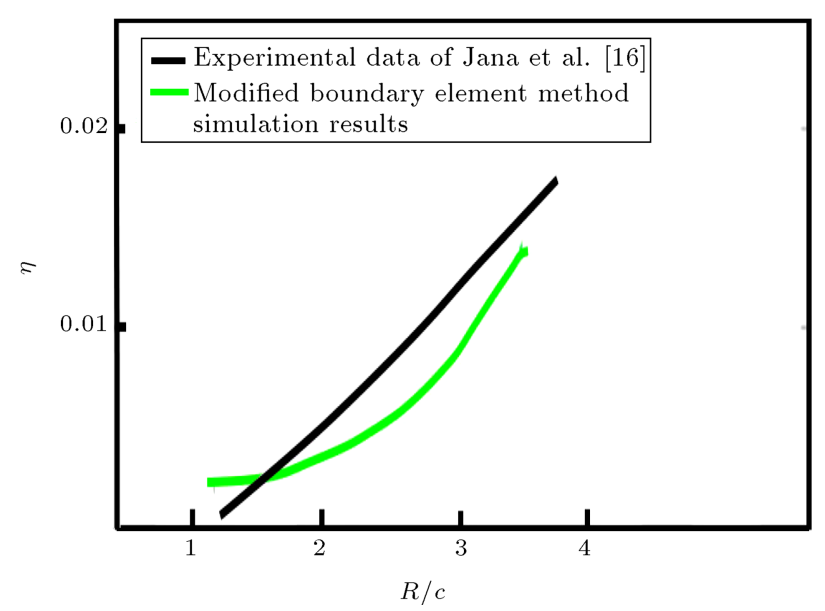

Figure 9. Swimming efficiencies of various trajectories followed by anterior and posterior portions of Paramecium compared to swimming efficiency obtained from numerical simulation. In numerical simulations, ratio of $R / c$ was varied from 1.3 to 3.6. Simulation results are in good agreement with experimental results of Jana et al. [16].

with less computational cost. In fact, the presented methodology is capable of coupling the internal mechanisms of cilia with external fluid dynamics, which is not only vital for investigating Paramecium locomotion, but may also be useful in determining the causes of cilia related diseases, such as primary cilia dyskinesia. For modeling Paramecium locomotion, its boundary 
was discretized into elements with lengths of $15 \mu \mathrm{m}$. This length was chosen because it enabled us to simulate Paramecium with hydrodynamically independent elements and a reduced computational cost. In fact, it is an inherent property of low-Reynolds fluid mechanics that hydrodynamic interactions are effective at low ranges. This is the key distinction of low-Reynolds swimming, which enables application of computational models with comparatively less computational cost. Hydrodynamical independence of adjacent elements is, of course, an approximation. However, results of the developed method depict that this approximation is more close to reality than analytical methods.

At each time step of swimming, the coordinates of each boundary element were extracted as a function of $Y$ (normal distance between each element and its corresponding wall) and $\theta$ (angle of rotation of element with respect to its corresponding wall). These two parameters, as well as the other parameters describing cilia coordinates (see Figure $\mathrm{S} 2$ and associated table in Supplementary Material (Section 6)), and the element velocities, were given to our numerical model for extracting the normal and tangential components of viscous forces applied onto each boundary element. By integrating these functions over the Paramecium boundary, propulsion force, swimming velocity and swimming trajectory were determined. The results of this study can be useful for determining Paramecium locomotion subjected to fluid motion within specific confinements. For example, data for a swimming trajectory can be useful in utilizing Paramecium as a micro robot for minimally invasive therapies.

\section{Supplementary materials}

Supplementary material of this article can be found at: https://jumpshare.com/v/pxrIRH8vmU2onBRN32rf

\section{Competing interests}

The authors declare that they have no competing interests.

\section{Acknowledgment}

The authors would like to thank members of the bioinspired fluid lab at Virginia Tech for experimental data and discussions.

\section{References}

1. Brennen, C. and Winet, H. "Fluid mechanics of propulsion by cilia and flagella", Ann. Rev. Fluid Mech., 9, pp. 339-398 (1977).
2. Machemer, H. "Ciliary activity and the origin of metachrony in Paramecium: effects of increased viscosity", Journal of Experimental Biology, 57, pp. 239-259 (1972).

3. Nelson, B.J., Kaliakatsos, I.K. and Abobott, J.J. "Microrobots for minimally invasive medicine", $A n$ nual Review Biomedical Engineering Aug., 15(12), pp. 55-85 (2010). Doi: 10.1146/annurev-bioeng-010510103409.

4. Lighthill, M.J. "On the squirming motion of nearly spherical deformable bodies through liquids at very small Reynolds numbers", Communications on Pure and Applied Mathematics, 5, pp.109-118 (1952).

5. Blake, J.R. "A spherical envelope approach to ciliary propulsion", Journal of Fluid Mechanics, 46, pp. 199208 (1971).

6. Krivovichev, G.V. and Tregoubov, V.P. "Mathematicall modelling of ciliary movement mechanism", Acta of Bioeng. and Biomech., 13(2), pp. 73-81 (2011).

7. Wang, S. and Ardekani, A. "Unsteady swimming of small organisms", Journal of Fluid Mechanics, 177, pp. 1-12 (2012).

8. Giacche, D. and Ishikawa, T. "Hydrodynamic interaction of two unsteady model microorganisms", Journal of Theoretical Biology, 267, pp. 252-263 (2010).

9. Michelin, S. and Lauga, E. "Efficiency optimization and symmetry-breaking in a model of ciliary locomotion", Physics of Fluids, 22, p. 111901 (2010).

10. Michelin, S. and Lauga, E. "Optimal feeding is optimal swimming for all Peclet numbers", Physics of Fluids, 23, p. 101901 (2011).

11. Zhu, L., Lauga, E. and Prandtl, L. "Low Reynolds swimming in a capillary tube", Journal of Fluid Mechanics, 726, pp. 285-311 (2013). Doi: $10.1017 /$ jfm.2013.225.

12. Gueron, S. and Liron, N. "Ciliary motion modeling, and dynamic multi cilia interactions", Biophysical Journal, 63, pp. 1045-1058 (1992).

13. Pak, O.S. and Lauga, E. "Theoretical models in low-Reynolds number locomotion", RSC Soft Matter No. 1. Fluid-structure Interactions at Low Reynolds Numbers (2012).

14. Gueron, S. and Gurevich, K.L. "Computation of the internal forces in cilia. Application to ciliary motion the effect of viscosity and cilia interactions", Biophysical Journal, 53, pp. 1658-1676 (1998).

15. Vilfan, A. and Julicher, F. "Hydrodynamic flow patterns and synchronization of beating cilia", Physical Review Letters, 96, 058102 (2006).

16. Jana, S., Um, H.S. and Jung, S. "Paramecium swimming in capillary tube", Physics of Fluids, 24, 041901 (2012). Doi: 10.1063/1.4704792.

17. Yang, X., Dillon, R.H. and Fauci, L.J. "An integrative computational model for multicilary beating", Bulletin of Mathematical Biology, 70, pp. 1192-1215 (2008). 
18. Ishikawa, T. and Hota, M. "Interaction of two swimming Paramecia", The Journal of Experimental Biology, 209, pp. 4452-4463 (2006).

19. Pozrikidis, C., A Practical Guide to Boundary Element Methods with the Software Library BEMLIB, 1st Edn. CRC Press (2002).

20. Lauga, E. "Locomotion in complex fluids: Integral theorems", Physics of Fluids, 26, p. 081902 (2014).

21. Riley, E.E. "Enhanced active swimming in viscoelastic fluids", EPL, 108, 34003 (2014).

22. Varade, V. et al. "Velocity measurement in low Reynolds and low Mach number slip flow through a tube", Experimental Thermal and Fluid Science, 60, pp. 284-289 (2015).

23. Liopis, I. and Pagonabarraga, I. "Hydrodynamic interactions in squirmer motion: Swimming with a neighbor and close to a wall", Journal of Non-Newtonian Fluid Mechanics, 165, pp. 946-952 (2010).

24. Jiang, H. et al. "Force-moment line element method for stokes flow around a slender body", Engineering Analysis with Boundary Elements, 44, pp. 120-129 (2014).

25. Ishimoto, K. and Gaffney, E.A. "A study of spermatozoan swimming stability near a surface", Journal of Theoretical Biology, 360, pp. 187-199 (2014).

\section{Biographies}

Ali Nematollahi Sarvestani received his MSc degree from Sharif University of Technology, Tehran, Iran. His research interest includes fluid flow simulation with biological applications. He has participated in IMECE a number of times and has performed research into finite element simulations, bioengineering and micro air vehicles.

Amir Shamloo received his $\mathrm{PhD}$ degree from Stanford University, CA, USA, in 2010. He is now Assistant Professor in the Mechanical Engineering Department of Sharif University of Technology, Tehran, Iran. His main research interest involves the design and fabrication of microfluidic devices and their application in biology, as well as simulating biological processes.

Mohammad Taghi Ahmadian is Professor in the Department of Mechanical Engineering at Sharif University of Technology, Tehran, Iran. His articles have been cited more than 2000 times and he is a reviewer for many scientific journals. His research mainly focuses on micro and nano sciences, specializing in dynamics and vibrations. 